\author{
Revista de Psicología Vol. 34 (1), 2016 (ISSN 0254-9247) \\ http://dx.doi.org/10.18800/psico.201601.005
}

\title{
Estudo correlacional: criatividade verbal e personalidade segundo Modelo dos Cinco Grandes Fatores em estudantes brasileiros $^{1}$
}

\author{
Tatiana de Cássia Nakano², Priscila Zaia ${ }^{3}$ e Karina da Silva Oliveira ${ }^{4}$ \\ PUC-Campinas, Brasil
}

\begin{abstract}
No Brasil, a escassez de estudos que investigam a relação entre os construtos da Criatividade e Personalidade, principalmente considerando-se o modelo Big Five, pode ser notada. Neste cenário, a partir da aplicação dos testes Pensando Criativamente com Palavras de Torrance (versão brasileira) e a Bateria Fatorial da Personalidade, o presente trabalho pesquisou em uma amostra de 83 estudantes brasileiros do Ensino Médio, com idades entre 14 e 18 anos $(M=16.01, D P=.95)$ a relação entre os construtos referidos anteriormente. Os resultados indicaram a existência de correlaçóes significativas apenas entre o fator Socialização e os dois índices criativos verbais (ICVI = .26; ICVII = .26), de modo a apontar que, nesta pesquisa, os construtos apresentam-se pouco relacionados. Discute-se a influência dos instrumentos e do modelo teórico adotado.
\end{abstract}

Palavras-chave: traços de personalidade, correlação estatística, aptidão, criatividade verbal.

Correlational study: verbal creativity and personality according to the Big Five Model in Brazilian students

In Brazil, few studies are found investigating the relationship between creativity and personality constructs, especially considering the Big Five Model. This study investigated a sample of 83 Brazilian high school students, between ages 14 and 18 years $(M=16.01$, $S D=0.95)$ that answered two questionnaires, the Torrance Thinking Creatively with Words (Brazilian version) and the Bateria Fatorial de Personalidade (Factorial Battery of Personality).

As autoras agradecem ao CNPQ e à Capes pelo apoio recebido.

2 Docente do curso de pós-graduação stricto sensu em Psicologia da Pontifícia Universidade Católica de Campinas. Contato: tatiananakano@hotmail.com

3 Doutoranda em Psicologia pela Pontifícia Universidade Católica de Campinas. Contato: priscilazaia@gmail.com

4 Doutoranda em Psicologia pela Pontifícia Universidade Católica de Campinas. Endereço postal: Departamento de Pós-Graduação em Psicologia da PUC-Campinas. Avenida John Boyd Dunlop, s/n. Jardim Ipaussurama - Campinas / SP, Brasil. CEP 13060-904. Contato: karina_oliv@yahoo.com.br 
The results indicated significant correlations only between the socialization factor and two verbal creative indexes $(\mathrm{ICVI}=.26$; ICVII $=.26)$. In the present study the investigated constructs are weakly related. The influence of the instrument and the theoretical model adopted in the findings is discussed.

Keywords: personality traits, statistical correlation, aptitude, verbal creativity.

Estudio correlacional: creatividad verbal y personalidade según el Modelo de Cinco Factores en estudiantes brasileros

En Brasil es escasa la literatura científica acerca de la relación entre los constructos de creatividad, personalidad y el Modelo de los Cinco Factores. A partir de la aplicación del Test de Pensamiento Creativo con Palabras de Torrance (versión brasilera) y la Batería Factorial de Personalidad en 83 estudiantes brasileros de secundaria, con rango de edad de entre 14-18 ańos $(M=16 ; D S=.95)$, se estudia la relación entre la creatividad verbal y la personalidad. Los resultados revelan la existencia de relaciones significativas bajas entre el factor socialización y dos índices creativos verbales (ICV I $=.26$; ICVII $=.26)$. Se discute la influencia del instrumento y del modelo teórico asumido en los hallazgos.

Palabras clave: rasgos de personalidad, correlación estadística, aptitud, creatividad verbal. 
Durante décadas de estudo da criatividade, destaque tem sido dado à investigação das características de personalidade presentes nas pessoas criativas, sendo este um dos aspectos que mais tem atraído a atenção dos pesquisadores da área (Stein, 1974). Dada sua influência no funcionamento criativo (Sternberg \& Lubart, 1996; Torrance \& Ball, 1990; Wallack \& Kogan, 1965; Wechsler, 2008), a combinação entre atributos de personalidade e a capacidade de pensar de forma criativa e inovadora tem sido apontado como um importante recurso de enfrentamento dos desafios vividos na sociedade atual (Alencar \& Fleith, 2008). Tal relação foi foco da pesquisa aqui apresentada.

A investigação de características de personalidade que podem explicar e prever as realizaçóes criativas tem sido foco de estudos científicos, objetivando aproximar dois campos com grande importância para a literatura científica (Mussel, Mckay, Ziegler, Hewig \& Kaufman, 2015). Tenta-se, dessa maneira, verificar a existência de atributos gerais envolvidos na chamada personalidade criativa, compreender se a personalidade de tais indivíduos difere em termos de características e, ainda, analisar como cada um dos cinco fatores da personalidade relaciona-se com a criatividade (Chávez-Eakle, Eakle \& Cruz-Fuentes, 2012; Christensen, Drewsen \& Maal, 2014). Sem tentar determinar uma relação de causa e efeito, a literatura tem demonstrado efeitos mútuos entre os construtos, visto que características de personalidade podem influenciar diretamente na realização criativa dos indivíduos e o potencial criativo pode direcionar o indivíduo a encontrar maneiras diferentes e inovadoras de melhorar sua personalidade (Halpin, Halpin \& Torrance, 1974). Desse modo os estudos têm sido direcionados em termos de quais seriam os atributos pessoais presentes nas pessoas eminentemente criativas, na tentativa de se compreender como se combinam características cognitivas, motivacionais e de personalidade nesses indivíduos (Alencar, 2007; Candeias, 2008). 
Como resultado de uma série de estudos conduzidos, notadamente no contexto internacional desde a década de 1950 (Feist, 1998), algumas descriçôes da personalidade criativa passaram a ser apontadas na literatura (Haller \& Courvoisier, 2010; Lubart, 2007), compostas por um conjunto relativamente estável de características (Barron \& Harrington, 1981). Considerando-se que o completo desenvolvimento do potencial criativo ocorrerá a partir do momento que for possível entender diferenças individuais dos sujeitos criativos (Smith, 2008), diversas investigações desenvolvidas permitiram que alguns traços de personalidade comumente encontrados nesses indivíduos pudessem ser isolados (Haller \& Courvoisier, 2010). Nesse sentido, destacam-se, de forma bastante consensual, a fluência, flexibilidade, originalidade, abertura à experiência, disponibilidade para correr riscos, independência de pensamento e julgamento, dentre outros, salientadas por importantes autores na área da criatividade (Guilford, 1967; Romo, 2008; Torrance, 1966; Torrance \& Ball, 1990; Torrance \& Safter, 1999; Wallach \& Kogan, 1965; Wechsler, 2008).

Ao se discutir a relaçáo entre os construtos, torna-se importante destacar a complexidade de ambos e a variedade de modelos teóricos disponíveis na literatura científica. Considerando-se que os avanços científicos na teoria da personalidade e sua medida podem resultar em uma falta de comparabilidade entre os vários tipos de medidas e de avaliaçóes disponíveis (Hampson \& Goldberg, 2006), sendo a mesma situação notada em relaçáo à criatividade (Beghetto, Plucker \& MaKinster, 2001; Cropley, 2000; Kim, 2011; Silvia, 2011; Treffinger, 2009), torna-se essencial esclarecer as bases teóricas que foram utilizadas no estudo aqui apresentado. Sabe-se que, embora haja uma variedade de definiçóes para cada construto, a avaliação irá depender da teoria adotada pelo pesquisador, de maneira que a forma como cada teoria conceitua o temo acaba por definir as principais características de cada posição teórica.

Assim, esclarece-se que a definiçáo de personalidade assumida baseia-se no Big Five Model, cuja importância ampara-se no fato de que as pesquisas em personalidade ganharam novo ímpeto e direção 
a partir do estabelecimento de um consenso acerca da sua estrutura, através desse modelo (Prinzie, Dekovic, Rejintjes, Stams \& Belsky, 2009). Ele tem se mostrado predominante na atualidade (Cuperman \& Ickes, 2009; Lang, John, Lüdtke, Schupp \& Wagner, 2011) e o mais apropriado para descrever a estrutura da personalidade, sobretudo da personalidade adulta do ponto de vista psicométrico (Garcia, 2006). A importância desse modelo embasa-se principalmente no fato de ele ter sido aplicado em diversas amostras, em diversas culturas e por meio de numerosas fontes de informação (incluindo autoavaliação, avaliação por pares e avaliações clínicas), tendo demonstrado sua adequação nesses diferentes usos (Costa \& Widiger, 1993; McCrae, 1993; Urquijo, 2001), independentemente do país, dos instrumentos de medição utilizados e da pessoa avaliada (Furnham \& Fudge 2008). O modelo representa uma avaliação empírica na área da personalidade (Gosling, Rentfrow \& Swann, 2003), visto que fornece uma descrição objetiva, consistente e replicável das dimensões da personalidade humana.

Nele, as diferenças individuais mais importantes podem ser organizadas em cinco grandes domínios, responsáveis pela maior parte da variação da personalidade humana (Digman, 1997; Dobewall, Aavik, Konstabel, Schwartz \& Realo, 2014; Roivainen, 2015; Soto \& John, 2009a). Os cinco domínios gerais são: Extroversão, Amabilidade, Conscienciosidade, Neuroticismo e Abertura à Experiência, os quais tornam possível caracterizar e diferenciar os indivíduos através dos padróes de comportamentos que apresentam (DeYoung, 2015; McCrae, 2008), de modo a permitir a junção de opiniōes e visões da personalidade a partir de um único modelo (John, Naumann \& Soto, 2008; McCrae \& John, 1992; Nunes \& Hutz, 2007; Soto \& John, 2009b). Nessa perspectiva, faz uso de um modelo geral composto por dois níveis hierárquicos, o qual emprega, em um primeiro nível, cinco amplos fatores, e, em um segundo nível, várias outras características, denominadas facetas (DeYoung, Quilty \& Peterson, 2007; Gosling et al., 2003).

A criatividade, por sua vez, tem sido definida como um processo de compreensão, por parte do indivíduo, de lacunas, falhas ou problemas em situaçóes cotidianas. A partir desta percepção existe a possibilidade 
de elaborar suposiçóes ou soluções, avaliar como tais propostas poderão ajudar a sanar as lacunas, optar por uma destas hipóteses e, por fim, iniciar o processo de resolução e comunicação de resultados (Hébert, Cramond, Neumeister, Millar \& Silvian, 2002; Shaughnessy, 1998; Torrance, 1963). Neste sentido, Jauk, Benedek, Dunst e Neubauer (2013) afirmam a existência de diferenças individuais na criatividade, as quais têm sido alvo de um importante número de pesquisas.

As abordagens atuais definem esse construto, considerando quatro categorias de investigação: a pessoa, processo, produto e ambiente criativo. Esta sistematização, originária de Rhodes (1961) e atualmente já quase universalizada (Morais, 2001) é conhecida como esquema dos 4P's (person, process, product e press). A dimensão que enfatiza a pessoa criativa inclui trabalhos que valorizam as características pessoais observáveis ou subjacentes no sujeito e inclui "estudos referentes às habilidades cognitivas, traços de personalidade, motivação, estilos de aprendizagem e estilos de criatividade" (Alencar, Bruno-Faria \& Fleith, 2010, p. 12), assim como fisiologia, temperamento, atitudes pessoais, hábitos, valores, processos mentais e atitudes emocionais (Kneller, 1971; Novaes, 1972). A importância do estudo dos traços e características de personalidade da pessoa criativa ampara-se na os benefícios advindos desse conhecimento, os quais podem ser usados na elaboração de programas que objetivam o desenvolvimento dessas habilidades, permitindo ainda a descoberta de barreiras que impedem a sua expressão, de forma a aumentar as possibilidades da manifestação criativa em todos os indivíduos (Stein, 1974). A pessoa criativa e suas características foi tomada como base na investigação aqui relatada.

Uma revisão de pesquisas realizadas com o objetivo de integrar personalidade (de uma forma geral, sem considerar o modelo teórico adotado) e criatividade encontrados na literatura científica internacional apontou para a existência de estudos voltados ao conhecimento de características associadas aos dois construtos. Como exemplos pode ser citado o estudo desenvolvido por Acar e Runco (2015) visando a investigação da relação entre personalidade e pensamento divergente, cujos resultados apontaram para correlaçóes positivas entre pensamento 
divergente e originalidade, de modo que aqueles indivíduos que possuem atitudes e valores relacionados à originalidade apresentaram maior propensão a pensar de maneira divergente. Também Martinsen (2011) relatou que os traços de personalidade mais relacionados ao desenvolvimento criativo seriam são independência, dominância, introversão, abertura aos estímulos, intuição, flexibilidade, neuroticismo, ambiguidade, perseverança, abertura a novas experiências, vontade de assumir riscos e independência de julgamento.

No Brasil, duas pesquisas foram encontradas. A primeira, desenvolvida por Fleith e Alencar (2008) teve como foco verificar a existência de características de personalidade e de fatores ambientais no desenvolvimento da criatividade em crianças de 8 a 12 anos. Os resultados mostraram que criatividade, autoconceito e clima em sala de aula possuem correlaçóes positivas entre si indicando que tanto fatores ambientais quanto pessoais podem promover o potencial criativo. Por sua vez, Nakano e Castro (2013) investigaram a relação entre criatividade e temperamento em 142 crianças e adolescentes de 11 a 14 anos. Os resultados mostraram que duas dimensóes do temperamento relacionaram-se com o desempenho criativo dos participantes: extroversão e intuição. Outro tipo de estudo refere-se à investigação da relação entre os construtos considerando-se o Modelo dos Cinco Grandes Fatores (CCF), cujos resultados têm destacado que, dentre esses, abertura à experiência tem sido comumente apontada nos estudos como o fator de personalidade que mais estaria associado à expressão criativa, tanto no contexto internacional (Batey, Chamorro-Premuzic \& Furnham, 2009; Batey \& Furnham, 2006; Batey, Furnham \& Saffiulina, 2010; Cheng, Kim \& Hull, 2010; Conner \& Silvia, 2015; Feist, 1998; Ivcevic \& Mayer, 2007; Nelson \& Rawlings, 2010; Silvia, Martin \& Nusbaum, 2009; Von Stumm, Chung \& Furnham, 2011), quanto no brasileiro (Pereira, 2006).

No entanto, o que se tem visto é que, dependendo do instrumento utilizado, as relaçóes se alteram. Batey e outros (2010), por exemplo, fazendo uso de um inventário encontraram correlaçóes significativas com abertura a experiências. Entretanto, quando uma escala de auto 
avaliação da criatividade é utilizada, os resultados apontaram para relações positivas e significativas com Extroversão e Abertura e relações negativas com Neuroticismo. Outros estudos também apresentaram relaçôes diferentes entre criatividade e os demais fatores da personalidade: correlação negativa da criatividade com socialização (Batey \& Furnham, 2006; Von Stumm et al., 2011) e correlação positiva com Extroversão (Furnham \& Bachtiar, 2008).

Ao analisar os estudos citados pode-se notar que diferentes resultados têm sido relatados na literatura científica nacional e internacional. Dependendo do instrumento utilizado e do modelo teórico envolvido, as relaçóes entre criatividade e personalidade se alteram. Também foi possível verificar que os resultados do trabalho empírico dos últimos anos apontam para a existência de uma consistente associação entre os dois construtos, ainda que importantes diferenças possam ser encontradas (Feist, 1998). A partir de tais constataçóes e da inexistência de estudos brasileiros que tenham como foco a relação entre criatividade e personalidade tomando-se como base o modelo dos Cinco Grandes Fatores, o objetivo do presente estudo é investigar a relação entre estes dois construtos em uma amostra de estudantes.

\section{Método}

\section{Participantes}

A amostra deste estudo foi composta por 83 alunos, sendo 53 do sexo feminino, provenientes do $1^{\circ}$ ano $(\mathrm{n}=22), 2^{\circ}$ ano $(\mathrm{n}=27)$ e $3^{\circ}$ ano $(\mathrm{n}=34)$ do Ensino Médio de uma escola pública brasileira localizada no interior do Estado de São Paulo, selecionada por conveniência. Os participantes possuíam idades entre 14 e 18 anos $(M=16.01 ; D P=.95)$

\section{Medidas e instrumentos de medição}

a. Pensando Criativamente com Palavras de Torrance - versão brasileira (Wechsler, 2004): Instrumento composto por 6 atividades, sendo que as três primeiras são baseadas em uma mesma imagem. 
$\mathrm{Na}$ primeira atividade deve-se fazer perguntas acerca da imagem. $\mathrm{Na}$ segunda deve-se adivinhar possíveis causas para explicar a imagem e na terceira atividade deve-se adivinhar as consequências a partir do desenho. $\mathrm{Na}$ atividade quatro é apresentado um produto e deve-se acrescentar características para melhorá-lo. A quinta atividade consiste em inventar usos diferentes para um objeto e na última atividade deve-se fazer suposiçóes a partir de uma dada cena. $\mathrm{O}$ instrumento avalia oito características, pontuadas em cada atividade, sendo elas: Fluência, Flexibilidade, Elaboração, Originalidade, Expressão de Emoção, Fantasia, Perspectiva Incomum e Uso de Analogias e Metáforas. A soma de tais características dá origem a dois Índices Criativos Verbais (I e II) sendo que o primeiro agrupa apenas as quatro primeiras características, consideradas cognitivas e o segundo agrupa a soma das oito (cognitivas e emocionais).

O teste encontra-se aprovado para uso profissional pelo Conselho Federal de Psicologia brasileiro. Os estudos de investigação das suas qualidades psicométricas foram conduzidos por Wechsler (2004). Evidências de validade concorrente foram investigadas, relacionando-se o desempenho dos sujeitos no instrumento e sua produção criativa na vida real, considerando-se um grupo critério de pessoas reconhecidamente criativas que receberam premiações em suas áreas de destaque $(n=59)$ e outro grupo considerado não criativo ou regular, grupo controle $(n=69)$. Os resultados apontaram que seis das oito características obtiveram correlaçóes significativas com a produção reconhecida do indivíduo (com exceção de Fantasia e Analogias), sendo que todas mostraram-se relacionadas com a produção total (considerando-se produçáo reconhecida e não reconhecida). Um segundo estudo, comparando o desempenho dos dois grupos indicou a existências de evidências de validade de critério, ao apontar diferenças significativas entre eles na maior parte das características criativas avaliadas pelo instrumento (novamente com exceçáo de Fantasia e Analogias).

A precisão foi investigada de duas maneiras. A primeira através do teste e reteste, em 53 participantes, realizado 15 dias após a primeira aplicação, indicou correlaçóes entre .31 e $.83(p \leq .02 ; p \leq .000)$ para 
todas as medidas avaliadas. A segunda, realizada por meio da análise da precisão dos juízes no processo de correção do teste (em um total de 30 estudantes ao longo de seis anos de investigação), demonstrou índices de correlação entre o juiz ideal e os demais acima de .90 para a maior parte das características criativas verbais, quando corrigidos 20 testes comuns a todos. A normatização do instrumento, por sua vez, foi realizada a partir de uma amostra composta por 879 estudantes de Ensino Médio e Superior. Os resultados mostraram influência das variáveis sexo e nível de ensino no desempenho dos participantes, de modo que as tabelas de correção são divididas de acordo com esses critérios.

b. Bateria Fatorial da Personalidade (Nunes, Hutz \& Nunes, 2010): Instrumento de autorrelato para avaliação da personalidade, brasileiro, baseado no Modelo dos Cinco Fatores (CCF), composto por 126 itens a serem respondidos dentro de uma escala Likert de 7 pontos. Abrange os seguintes fatores e subfcetas: Neuroticismo (N1 = Vulnerabilidade, N2 = Instabilidade Emocional, N3 = Passividade / Falta de Energia, N4 = Depressão), Extroversão (E1 = Comunicação, E2 = Altivez, E3 = Dinamismo, E4 = Interaçôes Sociais), Socialização (S1 = Amabilidade, S2 = Pró-sociabilidade, S3 = Confiança nas pessoas),

Realização (R1 = Competência, R2 = Ponderação / Prudência, R3 = Empenho / Comprometimento), Abertura à Experiência (A1 = Abertura a Ideias, A2 = Liberalismo, A3 = Busca por novidades).

Evidências de validade e precisão foram encontradas a partir de estudos realizados por Nunes, Hutz e Nunes (2010). Os estudos de busca por evidências de validade foram realizados a partir da aplicação da BFP junto a outros instrumentos. Os resultados das evidências de validade divergente apontaram para correlaçóes entre .10 e .22 com inteligência, entre .14 e .52 com interesses profissionais, entre .19 e .62 com dificuldades de escolha profissional, entre -.09 e .45 com autoeficácia para atividades ocupacionais e de 0,44 com bem-estar subjetivo. Estudos de validade de critério foram conduzidos com grupo controle e grupo critério (dependentes químicos) mostrou que os cinco fatores apontaram diferenças significativas entre os grupos. Os estudos convergentes com um teste de personalidade, ansiedade, depressão e 
autoestima indicaram correlaçóes positivas e significativas entre medidas semelhantes. A precisão foi calculada a partir da consistência interna pelo Alfa de Cronbach, e os resultados apontaram que a maioria das facetas apresentaram consistência interna acima de .60. As normas são apresentadas de acordo com uma tabela geral, tabela por sexo e tabela por Estado brasileiro.

\section{Procedimento e processamento de informação}

Primeiramente a pesquisa foi aprovada pelo Comitê de Ética em Pesquisa da PUC-Campinas. Os testes foram aplicados de forma coletiva em sala de aula somente naqueles estudantes cujos pais ou responsáveis assinaram o termo de consentimento livre e esclarecido, em aplicação única, com duração aproximada de 1 hora e 30 minutos. Os estudantes responderam primeiramente ao instrumento de criatividade, dado o fato do mesmo ter tempo controlado para resposta, seguido do instrumento de personalidade, sem limite de tempo.

\section{Resultados}

Inicialmente a estatística descritiva foi estimada, para todas as medidas dos dois instrumentos utilizados. Os resultados referentes à avaliação da criatividade encontram-se descritos na Tabela 1 e os de personalidade na Tabela 2.

Os resultados da Tabela 1 mostram que, em média, os participantes forneceram cerca de 53 respostas nas seis atividades (média de 8,8 respostas por atividade), informação que pode ser visualizada por meio da pontuaçáo em Fluência, definida como o número de respostas adequadas elaboradas pelo sujeito. A importância dessa característica ampara-se na constatação de que, quanto mais ideias tivermos, maiores seráo as possibilidades de conseguirmos soluçóes que solucionem o problema (Nakano, 2012). Em termos comparativos, a media alcançada pelos participantes da pesquisa assemelha-se àquela obtida durante o processo de validação do teste $(M=57.68)$ pelo grupo considerado náo criativo. 


\section{Tabela 1}

Estatistica descritiva (médias e desvio padrão para as medidas de criatividade)

\begin{tabular}{lcc}
\hline Medida & $M$ & $D P$ \\
\hline Fluência & 52.96 & 21.59 \\
Flexibilidade & 25.62 & 7.72 \\
Elaboração & 16.56 & 9.41 \\
Originalidade & 27.79 & 14.91 \\
Expressão de Emoção & 1.04 & 1.48 \\
Fantasia & 3.63 & 3.98 \\
Perspectiva Incomum & 2.09 & 2.13 \\
Analogias / Metáforas & 4.59 & 2.76 \\
ICV_1 & 123.20 & 46.30 \\
ICV_2 & 134.53 & 50.71 \\
\hline
\end{tabular}

Nota: $\mathrm{ICV} \_1$ = índice criativo verbal $1 ; \mathrm{ICV} \_2$ = índice criativo verbal 2

No entanto, faz-se notar ainda que muitas dessas ideias consistem em conteúdo repetido (visto que a média de pontuação em Flexibilidade, definida como a capacidade de produzir ideias variadas e pertencentes a diferentes categorias, cai para 25). Assim, pode-se verificar que as respostas nem sempre atendem ao requisito de serem diversificadas, de modo a demonstrar certa tendência a ver o problema sob restritos e limitados pontos de vista (Nakano, Wechsler \& Primi, 2011) visto que, das 53 idéias, mais da metade acabam por serem classificadas dentro da mesma categoria de resposta, sendo consideradas repetidas e, portanto, não pontuadas nessa característica. Quadro semelhante é observado em relação à pouca adição de detalhes nas respostas (pontuação em Elaboração) e em relação à sua raridade (pontuação em Originalidade). Novamente vê-se que a mesma situação foi notada pela autora do manual (Wechsler, 2004). É possível notar ainda média de pontuação muito baixa nas características emocionais da Criatividade (Expressão de Emoção, 
Fantasia, Perspectiva Incomum, Analogias / Metáforas). Nesse sentido, Nakano e outros (2015) verificaram, em seu estudo, um desempenho criativo bastante baixo obtido pela maior parte da amostra. A partir da análise dos itens via Teoria de Resposta ao Item, os autores concluíram acerca de uma distancia bastante grande entre o nível de habilidade apresentado pelos participantes e o nível requerido para se pontuar nas principais características criativas. Fez-se notar maior facilidade dos sujeitos em obter pontos nas características cognitivas da criatividade do que naquelas consideradas emocionais, tal como o resultado aqui encontrado. É importante destacar que essa situação é bastante comum em testes de criatividade, visto que os itens/respostas consideradas criativas representam expressóes mais raras, de modo a gerar distribuiçóes assimétricas (Silvia, Wigert, Reiter-Palmon \& Kaufman, 2012).

A partir dos resultados apresentados na Tabela 2 observa-se que, dentre os cinco grandes fatores, a maior média atingida pelos participantes foi no fator Realização (4.63 de um máximo possível de 7.00), o qual descreve características como grau de organização, persistência, controle e motivação (Nunes, Hutz \& Nunes, 2010). Considerando-se a tabela normativa da amostra geral, apresentada no manual do instrumento, verifica-se que tal média equivale a um percentil 30, interpretado pelos autores como classificação média. Por outro lado, a média mais baixa alcançada pertence ao fator Neuroticismo, associado, ainda de acordo com os autores, às características emocionais das pessoas, referindo-se ao nível crônico de ajustamento e instabilidade emocional dos indivíduos. Convém salientar, no entanto, que, ainda que tal fator tenha apresentado, na amostra, menor valor, quando comparado com a tabela normativa do instrumento, representa um percentil 70, considerado fronteiriço entre médio e alto.

Com a finalidade de verificar a relaçáo entre os construtos criatividade e personalidade, atendendo o objetivo principal do estudo, a Correlação de Pearson foi empregada. Os dez indicadores de Criatividade Verbal foram correlacionados com os cinco grandes fatores da Personalidade e suas subfacetas, cujos resultados são apontados na Tabela 3. 


\section{Tabela 2}

Estatística descritiva (médias e desvio padrão para as medidas de personalidade)

\begin{tabular}{lll}
\hline Medida & $M$ & $D P$ \\
\hline NEUROTICISMO & 3.63 & 0.75 \\
N1 & 4.09 & 1.11 \\
N2 & 3.96 & 1.38 \\
N3 & 3.86 & 1.22 \\
N4 & 2.60 & 0.84 \\
\hline EXTROVERSÃO & 4.34 & 0.65 \\
E1 & 4.29 & 0.76 \\
E2 & 3.67 & 0.87 \\
E3 & 4.65 & 1.01 \\
E4 & 4.76 & 1.12 \\
\hline SOCIALIZAÇÃO & 4.18 & 0.46 \\
S1 & 5.51 & 0.82 \\
S2 & 3.29 & 0.64 \\
S3 & 3.73 & 0.75 \\
\hline REALIZAÇÃO & 4.63 & 0.67 \\
R1 & 4.94 & 0.89 \\
R2 & 4.34 & 1.01 \\
R3 & 4.62 & 1.14 \\
ABERTURA & 4.16 & 0.54 \\
A1 & 3.65 & 0.64 \\
A2 & 4.50 & 0.95 \\
A3 & 4.34 & 0.69 \\
\hline & & \\
\hline
\end{tabular}


Estudo correlacional: criatividade verbal e personalidade... / Nakano et al.

\section{Tabela 3}

Correlação de Pearson entre os indicadores de criatividade verbal e grandes fatores e subfacetas da personalidade

\begin{tabular}{lccccccccccc}
\hline & Flu & Flex & Elab & Orig & Emo & Fant & P.Inc. & A/M & ICV1 & ICV2 \\
\hline N & 0.03 & -0.03 & $0.23^{*}$ & -0.01 & 0.05 & 0.08 & 0.08 & 0.05 & 0.05 & 0.07 \\
E & 0.16 & 0.05 & -0.04 & 0.12 & 0.00 & -0.05 & 0.09 & 0.09 & 0.11 & 0.11 \\
S & $0.29^{* *}$ & 0.16 & 0.06 & $0.27^{*}$ & 0.09 & 0.001 & 0.14 & $0.22^{*}$ & $0.26^{*}$ & $0.26^{*}$ \\
R & 0.17 & 0.16 & 0.12 & 0.08 & 0.10 & -0.005 & 0.14 & 0.12 & 0.15 & 0.15 \\
A & 0.03 & 0.01 & 0.11 & 0.07 & 0.07 & -0.03 & 0.06 & 0.10 & 0.06 & 0.07 \\
E1 & 0.03 & -0.00 & -0.06 & 0.00 & -0.11 & -0.04 & 0.02 & 0.04 & 0.01 & -0.01 \\
E2 & 0.05 & -0.02 & 0.09 & 0.00 & 0.00 & -0.05 & 0.05 & -0.04 & 0.04 & 0.03 \\
E3 & 0.14 & 0.06 & -0.07 & 0.17 & -0.03 & -0.004 & 0.07 & 0.16 & 0.12 & 0.12 \\
E4 & 0.18 & 0.08 & -0.06 & 0.12 & 0.12 & -0.04 & 0.09 & 0.07 & 0.12 & 0.12 \\
S1 & 0.16 & 0.08 & -0.01 & 0.10 & 0.09 & 0.01 & 0.03 & 0.05 & 0.11 & 0.11 \\
S2 & 0.19 & 0.11 & 0.02 & $0.24^{*}$ & -0.00 & 0.02 & 0.04 & 0.20 & 0.19 & 0.19 \\
S3 & 0.20 & 0.11 & 0.11 & 0.18 & 0.08 & -0.03 & 0.18 & 0.18 & 0.19 & 0.19 \\
R1 & 0.16 & 0.17 & 0.00 & 0.13 & 0.06 & -0.15 & 0.14 & 0.17 & 0.14 & 0.13 \\
R2 & 0.03 & 0.06 & 0.09 & 0.02 & 0.02 & 0.18 & 0.09 & -0.01 & 0.05 & 0.07 \\
R3 & 0.14 & 0.10 & 0.13 & 0.02 & 0.12 & -0.05 & 0.05 & 0.10 & 0.12 & 0.11 \\
A1 & -0.06 & -0.03 & 0.15 & 0.00 & -0.04 & 0.04 & -0.05 & 0.00 & -0.01 & 0.00 \\
A2 & 0.06 & 0.07 & 0.15 & 0.10 & 0.00 & -0.17 & 0.13 & 0.10 & 0.10 & 0.09 \\
A3 & 0.04 & -0.03 & -0.08 & 0.02 & 0.19 & 0.12 & 0.02 & 0.09 & 0.00 & 0.03 \\
N1 & 0.07 & 0.03 & 0.17 & 0.01 & 0.00 & 0.06 & 0.11 & -0.06 & 0.08 & 0.08 \\
N2 & -0.06 & -0.14 & 0.14 & -0.12 & 0.02 & -0.09 & -0.06 & 0.14 & -0.0 & -0.06 \\
N3 & 0.02 & -0.00 & 0.16 & 0.05 & -0.03 & 0.14 & 0.09 & -0.05 & 0.06 & 0.07 \\
N4 & 0.09 & 0.07 & 0.13 & 0.03 & 0.19 & 0.15 & 0.11 & 0.11 & 0.09 & 0.12 \\
\hline & & & & & & & & & & \\
& & & 0.019 &
\end{tabular}

Nota: ${ }^{* *} p \leq .01 ;{ }^{*} p \leq .05$. Flu = fluência, Flex = flexibilidade, Elab = elaboração, Orig $=$ originalidade, Emo $=$ expressão de emoção, Fant $=$ fantasia, P.Inc. $=$ perspectiva incomum, $\mathrm{A} / \mathrm{M}$ = analogias e metáforas, ICV1 = índice criativo verbal 1, ICV2 = índice criativo verbal $2, \mathrm{~N}$ = neuroticismo, $\mathrm{E}=$ extroversão, $\mathrm{S}=$ socialização, $\mathrm{R}$ = realização e $\mathrm{A}=$ abertura. 
De uma forma geral, foi possível verificar um número bastante reduzido de correlaçóes entre as medidas de criatividade e personalidade, demonstrando, na presente pesquisa, uma baixa relação entre os construtos. Considerando-se inicialmente os cinco fatores gerais da Personalidade e os dois índices criativos verbais, pode-se verificar que as únicas correlações significativas e positivas observadas foram no fator Socialização, tanto com o índice que representa as características cognitivas da criatividade $(r=0.26, p \leq .01)$ quanto o índice que considera características cognitivas e emocionais $(r=0,26, p \leq .01)$. O mesmo fator ainda apresentou correlação significativa com características isoladas da Criatividade: Fluência $(r=0.29, p \leq .001)$, Originalidade $(r=0.27, p \leq .01)$ e Analogias / Metáforas $(r=0.22, p \leq .01)$.

É possível ainda notar que nenhum dos outros fatores da Personalidade mostrou-se correlacionado significativamente com a Criatividade, inclusive o fator de Abertura à Experiência, cuja relação era hipoteticamente esperada, dada a revisáo de literatura. Correlaçóes positivas e significativas entre Neuroticismo e Elaboração $(r=0.23, p \leq .01)$ e entre Pró-sociabilidade e Originalidade $(\mathrm{r}=.24, p \leq .01)$ também foram encontradas.

\section{Discussão}

A revisão da literatura internacional apontou que o fator Abertura inserido no Modelo dos Cinco Grandes Fatores da Personalidade (CCF) é o que possui características que mais se aproximam da Criatividade (Batey et al., 2009; Batey \& Furnham, 2006; Batey et al., 2010; Cheng et al., 2010; Feist, 1998; Ivcevic \& Mayer, 2007; Nelson \& Rawlings, 2010; Silvia et al., 2009; Von Stumm et al., 2011), porém, a presente pesquisa obteve resultados diferentes. Na tentativa de interpretar os achados, algumas reflexóes se tornam relevantes. A primeira delas refere-se ao fato de que o instrumento utilizado para avaliar personalidade na presente pesquisa difere dos utilizados nas pesquisas supracitadas, tendo em comum apenas a base teórica do modelo CCF. 
Considerando-se que resultados exatamente opostos são relatados por Batey e Furnham (2006) e Von Stumm e outros (2011), os quais encontraram correlaçóes significativas entre criatividade e socialização, porém no sentido negativo, oposto ao aqui relatado e a constatação de Batey e outros (2009), de que quando os instrumentos são alterados, a relação entre os construtos também pode ser modificada, uma hipótese levantada ampara-se na possibilidade de que os resultados, contraditórios, possam estar relacionados ao tipo de instrumento utilizado na avaliação dos construtos. A influência dessa variável merece ser melhor investigada posteriormente, fazendo-se uso de outros testes.

Uma outra questão, que envolve a ausência da correlação esperada entre criatividade e o fator de personalidade intitulado abertura à experiências, pode ser interpretada tomando-se um aspecto específico: o controle do tempo de execução das atividades. Nesse sentido, destaca-se o fato de que no teste Pensando Criativamente com Palavras o tempo para realização das atividades é controlado, questão que pode ter influenciado a relação entre criatividade e abertura, visto que, segundo Batey e outros (2009), indivíduos com altos índices neste fator de personalidade podem preocupar-se mais com a qualidade das respostas em oposição à quantidade e rapidez exigida em instrumentos que possuem controle de tempo. Como conseqüência acabam produzindo um número menor de respostas. Considerando-se que a fluência (número de respostas) é a característica que determina se as demais características criativas serão avaliadas, visto que ela considera a adequação ou não da resposta, sendo que, em caso dela não ser considerada em fluência, a resposta é descartada de qualquer pontuação posterior, tal fato pode ter exercido influência importante no resultado total do sujeito no teste. Uma investigação mais qualitativa realizada junto aos indivíduos com maior pontuação em abertura poderia fornecer indícios mais evidentes acerca da relação entre a pontuação deles na fluência (quantidade de idéias) e sua qualidade (pontuação nas demais características). Do mesmo modo, Conner e Silvia (2015) citam um estudo em que abertura também não se correlacionou com criatividade, apresentando como hipótese o fato de que o tamanho 
da amostra, limitado, pode ter interferido nos resultados. Essa é mais uma informaçáo que deve ser considerada ao observar os resultados da presente pesquisa, considerando que a amostra foi relativamente pequena, ficando abaixo de 100 indivíduos.

Tendo-se a hipótese, apontada anteriormente, de que o instrumento utilizado pode alterar a relação entre os construtos, uma segunda análise, mais minuciosa, foi feita considerando-se não os fatores que compóem o Big Five, mas sim os itens que fazem parte do instrumento utilizado. Para isso, cada um dos 126 itens da Bateria Fatorial de Personalidade foi, separadamente, correlacionado com as medidas totais de criatividade (ICVI e ICVII), visando-se identificar possíveis conteúdos relacionados entre os dois construtos. Os resultados mostraram correlaçôes significativas encontradas entre um item do fator Neuroticismo (item 124), três itens de Socialização (itens 61, 68 e 30) e dois itens de Realização (itens 122 e 114) com os índices criativos verbais. Novamente nenhum item dos fatores Extroversão e Abertura apontaram correlaçôes significativas com os índices criativos verbais.

Analisando-se o conteúdo presente nesses itens pode-se verificar que o item do fator Neuroticismo que se correlacionou significativamente com ambos os índices da criatividade $(r=.26 ; r=.28, p \leq .01$, respectivamente) envolve conteúdo relacionado à dificuldade do indivíduo em dormir. Nos itens de Socialização, um item esteve correlacionou-se positivamente com o índice criativo verbal II $(r=.21$, $p \leq .01)$, estando relacionado ao fato do indivíduo incentivar as pessoas à sua volta, outro item correlacionou-se positivamente com ambos os índices criativos $(r=.23$ e $r=0.22, p \leq .01)$, cujo conteúdo envolvia a confiança depositada naquilo que as pessoas dizem e, por fim, outro item relacionado às dúvidas que o indivíduo possui em relação às intençóes das pessoas apresentou correlação significativa com ambos os índices criativos $(r=.23$ para ambos os índices, $p \leq .01)$. Em relaçáo ao fator Realização, um item relacionado ao fato do indivíduo conseguir saber o que deseja para sua vida correlacionou-se negativamente com o índice criativo verbal II $(r=.21, p \leq .01)$ e um item relacionado à grande exigência que o indivíduo tem com ele mesmo 
correlacionou-se positivamente com ambos os índices criativos verbais $(r=.33, p \leq .01)$.

A análise do conteúdo abordado em cada um dos itens de personalidade que apresentou correlaçâo significativa com criatividade mostra que, aparentemente, a maior parte deles (dificuldade em dormir, duvidar das intençôes das pessoas, não saber o que deseja para a sua vida) não refletem as características tidas como típicas do indivíduo criativo, conforme revisão da literatura. No entanto, outros conteúdos, relacionados à atitudes tais como incentivar pessoas à sua volta, confiar nas pessoas, exigir demais de si mesmo concordam com as descrições fornecidas por importantes pesquisadores na temática (Guilford, 1967; Romo, 2008; Torrance, 1966; Torrance \& Ball, 1990; Torrance \& Safter, 1999; Wallach \& Kogan, 1965; Wechsler, 2008). Notadamente encontram-se relacionados às habilidades de autoeficácia, independência de julgamento, inconformismo e auto cobrança elevada (Wechsler, 2008).

Uma possível hipótese explicativa para os itens do fator Abertura do instrumento utilizado náo se correlacionarem significativamente com os índices criativos, indo em sentido contrário aos dados da literatura, pode referir-se ao fato de que, ao analisá-los em termos de conteúdo, foi possível observar que, apesar da definição do fator relacioná-lo à comportamentos exploratórios e de reconhecimento da importância de ter novas experiências, descrevendo os indivíduos com altos níveis nesse fator como pessoas criativas, curiosas e imaginativas (Nunes, Hutz \& Nunes, 2010), o conteúdo de tais itens não se relaciona diretamente à abertura caracterizada enquanto criatividade. Eles envolvem muito mais comportamentos relacionados ao ir além do tradicionalismo, de estar aberto à novas experiências cotidianas, desprendendo-se de padróes ou valores arraigados culturalmente, não correspondendo à definição de abertura enquanto característica criativa marcada por atitudes que envolvem curiosidade, uso da imaginação, fantasia e atividade criativa.

Como exemplo do tipo de conteúdo presente nos itens de abertura da bateria podem ser citadas afirmaçóes relacionadas a seguir regras sociais sem questionamento, pensar de forma diferente do que se pensava antigamente, modificar os caminhos feitos diariamente, ir a 
lugares desconhecidos, dentre outras. Tais definiçóes distanciam-se da definiçẫo de criatividade que embasa o instrumento de avaliação desse construto utilizado neste estudo, assim como da maior parte das definiçóes encontradas na literatura, as quais envolvem a compreensão das lacunas existentes e busca por soluçôes inovadoras de resolução de problemas cotidianos (Hébert et al., 2002; Shaughnessy, 1998; Torrance, 1963). Dado o distanciamento entre o conteúdo envolvido nos itens de abertura do instrumento de personalidade utilizado e da definiçáo de abertura enquanto característica criativa, a ausência de correlação significativa entre as medidas se torna justificável. Nesse sentido, outros estudos envolvendo diferentes instrumentos poderão confirmar ou não os dados aqui encontrados, de modo que a relação entre o fator de personalidade denominado abertura a idéias com a criatividade possa ser melhor esclarecida.

Ainda no que se refere à relação dos construtos é importante salientar que, apesar da existência de uma vasta gama de estudos referindo-se à relação da criatividade com o fator abertura, outros estudos que relatam a relação significativa entre criatividade e o fator socialização também são encontrados (Christensen et al., 2014; Hughes, Furnham \& Batey, 2013; Santos, Sisto \& Martins, 2003; Silvia et al., 2011), tal como os relatados aqui. Nesse sentido a literatura também tem destacado que a pesquisa neste campo pode beneficiar-se com a investigação mais ampla de todos os fatores de personalidade, porém, pelo fato do fator abertura possuir papel central na relaçáo com criatividade, em grande parte dos estudos, a maioria acaba por suprimir os demais fatores ou deixá-los em segundo plano, ignorando o fato de que seus resultados também possam contribuir para a melhor compreensão da expressão criativa (Silvia et al., 2011). Isso porque, ainda de acordo com os autores, o fator Socializaçáo torna-se intrigante pois conecta-se às características interpessoais da criatividade, as quais não têm sido muito investigadas, ao contrário daquelas voltadas ao aspecto cognitivo e comportamental do construto.

Considerando-se que a definição do fator Socialização na Bateria Fatorial da Personalidade explicita que os indivíduos com pontuação 
alta nesse fator apresentam maior preocupação e desejo em ajudar os demais, possuindo alto nível de altruísmo, relacionando-se diretamente ao desenvolvimento psicossocial das pessoas, à formação da sua identidade e da capacidade de interação com os demais (Nunes et al., 2010), pode-se verificar que dentre as características de uma personalidade criativa encontra-se a empatia, de maneira a sugerir a presença de características relacionadas às relaçôes interpessoais (Chávez-Eakle et. al., 2012). Tal constatação pode ser utilizada como base para a interpretação da correlação encontrada na presente pesquisa entre criatividade e Socialização. Outro ponto a ser destacado, segundo Csikszentmihalyi (1999) ampara-se no fato de que os produtos criativos não são produzidos por indivíduos isolados, mas por pessoas que estão inseridas em um ambiente e rodeados por outras. Como conseqüência, de acordo com o autor, qualquer definição de criatividade deve incorporar a importância das relaçóes interpessoais e da cultura visto que indivíduos que apresentem bem desenvolvidas características de criatividade e de socialização apresentariam tendência a elaborar soluções mais eficientes, sábias e aplicáveis, visto que os traços relacionados a este grande fator da personalidade fornecem, dentre outras habilidades, motivação pró-social como combustível para que os indivíduos solucionem as lacunas cotidianas (Myszkowski, Storme, Davila \& Lubart, 2015).

A presente pesquisa teve como objetivo identificar a relação entre dois construtos há muito tempo estudados pela literatura científica: criatividade e personalidade, buscando ainda proporcionar uma contribuição para a discussão da temática, a partir da percepção de lacunas relacionadas a este tipo de estudo presentes na literatura científica brasileira. A partir da análise dos resultados foi possível observar a existência de uma correlação significativa entre Criatividade e um dos grandes fatores da Personalidade (Socialização). A correlação encontrada não deve ser descartada, baseando-se na amplitude e importância dos fenômenos em questão, porém, recomenda-se cautela na interpretação dos resultados, considerando as limitaçóes do estudo. $\mathrm{O}$ tamanho da amostra e os instrumentos utilizados podem ter influenciado os dados aqui apresentados, de modo que a condução de novos estudos com 
variação de instrumentos, ampliação e diversificação da amostra são incentivados. Os resultados provenientes destes novos estudos poderão confirmar ou não tais dados, de maneira a contribuir para uma discussão mais completa acerca da relação entre Criatividade e Personalidade em amostras brasileiras.

Estudos que apresentem resultados similares ao da presente pesquisa são raros na literatura científica, visto que, geralmente, criatividade relaciona-se a abertura à experiência, porém, apesar disto, almeja-se que o conhecimento gerado a partir da relação encontrada entre Criatividade e Socialização possa alcançar novas maneiras de se olhar para estes dois fenômenos, considerando a amplitude de ambos e as influências culturais que englobam o fenômeno da criatividade, as quais devem ser consideradas tanto na definição do mesmo quanto na interpretação de resultados relacionados à ele.

\section{Referências}

Acar, S. \& Runco, M. A. (2015). Thinking in Multiple Directions: Hyperspace Categories in Divergent Thinking. Psychology of Aesthetics, Creativity, and the Arts, 9(1), 41-53. http://dx.doi. org/10.1037/a0038501

Alencar, E. M. L. S. (2007). Criatividade no contexto educacional: três décadas de pesquisa. Psicologia Teoria e Pesquisa, 23, no.spe, 45-49. http://dx.doi.org/10.1590/S0102-37722007000500008

Alencar, E. M. L. S. \& Fleith, D. S. (2008). Barreiras à promoção da criatividade no ensino fundamental. Psicologia: Teoria e Pesquisa, 24(1), 59-65. http://dx.doi.org/10.1590/s0102-37722008000 100007

Alencar, E. M. L. S., Bruno-Faria, M. F. \& Fleith, D. S. (Org.). (2010). Medidas de Criatividade. Porto Alegre: Artmed

Barron, F. \& Harrington, D. M. (1981). Creativity, Intelligence, and Personality. Annual Review of Psychology, 32(1), 439-476. http:// dx.doi.org/10.1146/annurev.ps.32.020181.002255 
Batey, M., Chamorro-Premuzic, T. \& Furnham, A. (2009). Intelligence and personality as predictors of divergent thinking: the role of general, fluid and crystallized intelligence. Thinking Skills and Creativity, 4, 60-69. http://dx.doi.org/10.1016/ j.tsc.2009.01.002

Batey, M. \& Furnham, A. (2006). Creativity, intelligence and personality: A critical review of the scattered literature. Genetic, General and Social Psychology Monographs, 132, 355-429. http:// dx.doi.org/10.3200/MONO.132.4.355-430

Batey, M., Furnham, A. \& Safiullina, X. (2010). Intelligence, general knowledge and Personality as predictors of creativity. Learning \& Individual Differences, 20(5), 532-535. http://dx.doi. org/10.1016/j.lindif.2010.04.008

Beghetto, R.A., Plucker, J.A. \& MaKinster, J.G. (2001). Who studies creativity and how do we know? Creativity Research Journal, 13(3/4), 351-357. http://dx.doi.org/10.1207/S15326 934CRJ1334_12

Candeias, A. A. (2008). Criatividade: perspectiva integrativa sobre o conceito e a sua avaliação. In M.F. Morais \& S. Bahia (Orgs.), Criatividade: conceito, necessidades e intervenção (pp. 41-64). Braga, Portugal: Psiquilíbrios.

Chávez-Eakle, R., Eakle, A. \& Cruz-Fuentes, C. (2012). The Multiple Relations Between Creativity and Personality. Creativity Research Journal, 24(1), 76-82. http://dx.doi.org/10.1080/10400419.20 12.649233

Cheng, Y., Kim,K.H. \& Hull, M.F. (2010). Comparisons of creative styles and personality types between american and taiwanese college students and the relationship between creative potential and personality types. Psychology of Aesthetics, Creativity and the Arts, 4(2), 103- 112. http://dx.doi.org/10.1037/a0017430

Christensen, B. T., Drewsen, L. K. \& Maal, J. (2014). Implicit Theories of the Personality of the Ideal Creative Employee. Psychology of Aesthetics, Creativity and the Arts, 8(2), 189-197. http://dx.doi. org/10.1037/a0036197 
Conner, T. S. \& Silvia, P. J. (2015). Creative Days: A Daily Diary Study of Emotion, Personality, and Everyday Creativity. Psychology of Aesthetics, Creativity, and the Arts. 10.1037/aca0000022 http:// dx.doi.org/10.1037/aca0000022

Costa, P. T. \& Widiger, T. A. (1993). Introduction. In P. T. Costa \& T. A. Widiger (Orgs.), Personality Disorders and the five-factor model of personality (pp. 1-10). Washington, DC: American Psychological Association.

Cropley, A. (2000). Defining and measuring creativity: are creativity tests worth using?. Roeper Review, 23, 72-79. http://dx.doi. org/10.1080/02783190009554069

Csikszentmihalyi, M. (1999) Creativity. In R. Sternberg (Ed.), Handbook of Creativity (pp. 313-330). Cambridge: Cambridge University Press.

Cuperman, R. \& Ickes, W. (2009). Big Five predictors of behavior and perception in initial dyatic interaction: personality similarity helps extraverts and introverts, but hurts "disagreeable". Journal of Personality and Social Psychology, 97, 667-684. http://dx.doi. org/10.1037/a0015741

DeYoung, C. G. (2015). Cybernetic Big Five Theory. Journal of Research in Personality, 56, 33-58. http://dx.doi.org/10.1016/ j.jrp.2014.07.004

DeYoung, C. G., Quilty, L. C. \& Peterson, J. B. (2007). Between Facets and Domains: 10 Aspects of the Big Five. Journal of Personality and Social Psychology, 93(5), 880-896. http://dx.doi. org/10.1037/0022-3514.93.5.880

Digman, J. M. (1997). Higher-Order Factors of the Big Five. Journal of Personality and Social Psychology, 73(6), 1246-1256. http:// dx.doi.org/10.1037/0022-3514.73.6.1246

Dobewall, H., Aavik, T., Konstabel, K., Schwartz, S. H. \& Realo, A. (2014). A comparison of self-other agrément in personal values versus the Big Five Personality traits. Journal of Research in Personality, 50, 1-10. http://dx.doi.org/10.1016/ j.jrp.2014.01.004 
Feist, G. J. (1998). A meta-analysis of personality in scientific and artistic creativity. Personality and Social Psychology Review, 4, 290-309. http://dx.doi.org/10.1207/s15327957pspr0204_5

Fleith, D. S. \& Alencar, E. M. L. S. (2008). Características Personológicas e Fatores Ambientais relacionados à Criatividade dos alunos do ensino fundamental. Avaliação Psicológica, 7(1), 35-44.

Furnham, A. \& Bachtiar, V. (2008). Personality and intelligence as predictors of creativity. Personality and Individual Differences 45, 613-617. http://dx.doi.org/10.1016/j.paid.2008.06.023

Furnham, A. \& Fudge, C. (2008). The five factor model of personality and sales performance. Journal of Individual Differences, 29, 11-16. http://dx.doi.org/10.1027/1614-0001.29.1.11

Garcia, L. F. (2006). Teorias psicométricas da personalidade. In C. E. Flores-Mendoza \& R. Colom (Orgs.), Introdução à psicologia das diferenças individuais (pp. 219-242). Porto Alegre: Artmed.

Gosling, S. D., Rentfrow, P. J. \& Swann, W. B. Jr. (2003). A very brief measure of the Big-Five Personality domains. Journal of Research in Personality, 37, 504-528. http://dx.doi.org/10.1016/S00926566(03)00046-1

Guilford, J. P. (1967). The nature of human intelligence. Nova York: McGraw-Hill.

Haller, C. S. \& Courvoisier, D. S. (2010). Personality and thinking style in different creative domains. Psychology of Aestetics, Creativity and the Arts, 4(3), 149-160. http://dx.doi.org/10.1037/a0017084

Halpin, G., Halpin, G. \& Torrance, E. P. (1974). Relationships between creative thinking abilities and measure of the creative personality. Education and Psychological Measurement, 34, 75-82. http://dx.doi.org/10.1177/001316447403400111

Hampson, S. E. \& Goldberg, L. R. (2006). A first large cohort study of personality trait stability over the 40 years between elementary school and midlife. Journal of Personality and Social Psychology, 91(4), 763-779. http://dx.doi.org/10.1037/00223514.91.4.763 
Hébert, T. P., Cramond, B., Neumeister, K. L. S., Millar, G. \& Silvian, A. F. (2002, February). E. Paul Torrance: His Life Accomplishments, and Legacy. The National Research Center on the Gifted and Talented. University of Connecticut. Recuperado dia 28 de março de 2014, de http://www.gifted.uconn.edu/nrcgt/reports/ rm02152/rm02152.pdf

Hughes, D. J., Furnham, A. \& Batey, M. (2013). The structure and Personality predictors of self-rated creativity. Thinking Skills and Creativity, 9, 76-84. http://dx.doi.org/10.1016/ j.tsc.2012.10.001

Ivcevic, Z. \& Mayer, J.D. (2007). Creative types and personality. Imagination, cognition and personality, 26(1-2), 65-86. http:// dx.doi.org/10.2190/0615-6262-G582-853U

Jauk, E., Benedek, M., Dunst, B. \& Neubauer A. C. (2013). The relationship between intelligence and creativity: New support for the threshold hypothesis by means of empirical breakpoint detection. Intelligence, 41, 212-221. http://dx.doi.org/10.1016/ j.intell.2013.03.003

John, O. P., Naumann, L. P. \& Soto, C. J. (2008). Paradigm Shift to the Integrative Big-Five Trait Taxonomy: History, Measurement, and Conceptual Issues. In O. P. John, R. W. Robins \& L. A. Pervin (Eds.), Handbook of Personality: Theory and Research, (3nd ed., pp. 114-158). New York, NY: Guilford Press.

Kim, K. H. (2011). The creativity crisis: the decrease in creative thinking scores on the Torrance Tests of Creative Thinking. Creativity Research Journal, 23 (4), 285-295 http://dx.doi.org/1 0.1080/10400419.2011.627805

Kneller, G.F. (1971). Arte e ciência da criatividade. São Paulo: Ibrasa.

Lang, F. R., John, D., Lüdtke, O., Schupp, J. \& Wagner, G. G. (2011).

Short assessment of the Big Five: robust across survey methods except telephone interviewing. Behaviour Research and Therapy, 43, 548-567. http://dx.doi.org/10.3758/s13428-011-0066-z Lubart, T. (2007). Psicologia da criatividade. Porto Alegre: ArtMed. 
Martinsen, O. L. (2011). The Creative Personality: A Synthesis and Development of the Creative Person Profile. Creativity Research Journal, 23(3), 185-202. http://dx.doi.org/10.1080/10400419. 2011.595656

McCrae, R. R. (1993). Openness to experience as a basic dimension of personality. Imagination, Cognition and Personality, 13, 39-55. http://dx.doi.org/10.2190/H8H6-QYKR-KEU8-GAQ0

McCrae, R. R. (2008). A role on some measures of profile agreement. Journal of Personality Assessment, 90, 105-109. http://dx.doi. org/10.1080/00223890701845104

McCrae, R. R. \& John, O. P. (1992). An introduction to the Five-Factor Model and its Applications. Journal of Personality, 60(2), 175215. http://dx.doi.org/10.1111/j.1467-6494.1992.tb00970.x

Morais, M.F. (2001). Definição e avaliação da criatividade. Braga: Universidade do Minho.

Mussel, P., Mckay, A. S., Ziegler, M., Hewig, J. \& Kaufman, J. C. (2015). Predicting Creativity Based on the Facets of the Theoretical Intellect Framework. European Journal of Personality. http://dx.doi.org/10.1002/per.2000

Myszkowski, N., Storme, M., Davila, A. \& Lubart, T. (2015). Managerial creative problem solving and the Big Five Personality traits: Distinguishing divergent and convergent Abilities. Journal of Management Development, 34(6), 674-684. http://dx.doi. org/10.1108/JMD-12-2013-0160

Nakano, T.C. (2012). O desenho na expressão criativa: teste de criatividade figural infantil. In S.M. Wechsler \& T.C. Nakano (Eds.), O desenho infantil: forma de expressão cognitiva, criativa e emocional (pp. 67-95). São Paulo: Casa do Psicólogo.

Nakano, T. C. \& Castro, L. R. (2013). Relação entre criatividade e traços temperamentais em estudantes do ensino fundamental. Psico-USF, 18(2), 249-262. http://dx.doi.org/10.1590/S141382712013000200009 
Nakano, T. C., Primi, R., Abreu, I. C. C., Gozzoli, M. Z., Caporossi, D. C., Miliani, A. F. M. \& Martins, A. A. (2015). Bateria para avaliação das altas habilidades/superdotação: análise dos itens via Teoria de Resposta ao Item. Estudos de Psicologia (Campinas), 32(4), 725-737. http://dx.doi.org/10.1590/0103-166 X2015000400016

Nakano, T.C., Wechsler, S.M. \& Primi, R. (2011). Teste de Criatividade Figural Infantil. São Paulo: Vetor.

Nelson, B. \& Rawlings, D. (2010). Relating schizotypy and Personality to the phenomenology of creativity. Schizophrenia Bulletin, 36(2), 388-399. http://dx.doi.org/10.1093/schbul/sbn098

Novaes, M. H. (1972). Psicologia da criatividade. Rio de Janeiro: Vozes.

Nunes, C. H. S. \& Hutz, C. S. (2007). Construção e Validação da Escala Fatorial de Socialização. Psicologia: Reflexão e Crítica, 20(1), 20- 25.

Nunes, C. H. S. S., Hutz, C. S. \& Nunes, M. F. O. (2010). Bateria Fatorial de Personalidade. Manual Técnico. Sáo Paulo: Casa do Psicólogo.

Pereira, D. F. (2006). Um estudo sobre o Wartegg como medida de criatividade em seleção de pessoal (Dissertação de Mestrado não publicada). Faculdade de Psicologia da Universidade Federal do Rio Grande do Sul, Porto Alegre, Rio Grande do Sul.

Prinzie, P. Dekovic, M. Reijntjes, A. H. A. Stams, G. J. J. M. \& Belsky, J. (2009). The Relations Between Parents' Big Five Personality Factors and Parenting: A Meta-Analytic Review. Journal of Personality and Social Psychology, 97(2), 351-362. http://dx.doi. org/10.1037/a0015823

Rhodes, M. (1961). An analysis of creativity. Phi Delta Kappa, 42, 305310.

Romo, M. (2008). Creatividad em los domínios artístico y cientifico y sus correlatos educativos. In M.F. Morais \& S. Bahia (Orgs.), Criatividade: conceitos, necessidades e intervençâo (pp. 65-90). Minho: Psiquilibrios. 
Santos, A. P. A., Sisto, F. F. \& Martins, R. M. M. (2003). Estilos cognitivos e personalidade: um estudo exploratório de evidências de validade. Psico-USF, 6(2), 55-64. http://dx.doi.org/10.1590/ s1413-82712003000100003

Shaughnessy, M. F. (1998). An Interview with E. Paul Torrance: About Creativity. Educational Psychology Review, 10(4), 441-452.

Silvia, P. J. (2011). Subjective scoring of divergent thinking: examining the reliability of unusual uses, instances and consequences tasks. Thinking Skills and Creativity, 6, 24-30. http://dx.doi. org/10.1016/j.tsc.2010.06.001

Silvia, P. J., Kaufman, J. C., Reiter-Palmon, R. \& Vigert, B. (2011). Cantankerous creativity: Honesty-Humility, Agreeableness, and the HEXACO structure of creative achievement. Personality and Individual Differences, 51, 687-689. http://dx.doi.org/10.1016/ j.paid.2011.06.011

Silvia, P.J., Martin, C. \& Nusbaum, E.C. (2009). A snapshot of creativity: evaluating a quick and simple method for assessing divergent thinking. Thinking Skills and Creativity, 4, 79-85. http://dx.doi.org/10.1016/j.tsc.2009.06.005

Silvia, P. J., Wigert, B., Reiter-Palmon, R. \& Kaufman, J.C. (2012). Assessing creativity with self-report scales: a review and empirical evaluation. Psychology of Aesthetics, Creativity, and the Arts, 6(1) 19-34. http://dx.doi.org/10.1037/a0024071

Smith, G. W. (2008). The Creative Personality in search of a theory. Creativity Research Journal, 20(4), 383-390. http://dx.doi. org/10.1080/10400410802391645

Soto, C. J. \& John, O. P. (2009a). Ten facet Scales for the Big Five Inventory: Convergence with NEO PI-R facets, self-peer agreement, and discriminant validity. Journal of Research in Personality, 43, 84-90. http://dx.doi.org/10.1016/j.jrp.2008.10.002

Soto, C. J. \& John, O. P. (2009b). Using the California Psychological Inventory to assess the Big Five Personality domains: A hierarchical approach. Journal of Research in Personality, 43, 25-38.

Stein, M. I. (1974). Stimulating creativity. Nova York: Academic Press. 
Sternberg, R. J. \& Lubart, T. I. (1996). Investing in creativity. American Psychologist, 51, 677-688. http://dx.doi.org/10.1037/0003-066 X.51.7.677

Torrance, E. P. \& Safter, H. T. (1999). Making the creative leap beyond. Buffalo, NY: Creative Education Foundation.

Torrance, E. P. (1963). Creativity in the Classroom: What Research Says to the Teacher. National Education Association, 37 p.

Torrance, E. P. (1966). Torrance tests of creative thinking. Lexington: Personnel Press.

Torrance, E. P., Ball, O. E. (1990). Streamlined scoring and interpretation guide and norms manual verbal and figural form $B$. Bensenville: Scholastic Testing Service.

Treffinger, D. J. (2009). Myth 5: creativity is too difficult to measure. Gifted Child Quartely, 53(4), 245-247. http://dx.doi.org/10. $1177 / 0016986209346829$

Urquijo, S. (2001). Modelos circumplexos da personalidade. In F. F. Sisto, E. T. B. Sbardelini \& R. Primi. (Orgs.), Contextos e questóes da avaliação psicológica (pp. 31-49). São Paulo: Casa do Psicólogo. Von Stumm, S., Chung, A. \& Furnham, A. (2011). Creative Ability, Creative Ideation and Latent Classes of Creative Achievement: What Is the Role of Personality?. Psychology of Aesthetics, Creativity, and the Arts. Advance online publication.

Wallack, M. \& Kogan, N. (1965). Modes of thinking in young children. Nova Iorque: Holt, Rinehart and Winston.

Wechsler, S. M. (2008). Criatividade: descobrindo e encorajando. Campinas: LAMP / Impressão Digital do Brasil.

Wechsler, S.M. (2004). Pensando Criativamente com Palavras - testes de Torrance (versão brasileira). Campinas: LAMP / Impressão Digital do Brasil.

Recibido: 25 de julio de 2015 Aceptado: 29 de septiembre de 2015 